\title{
COMPOSITION OPERATORS ON WEIGHTED BERGMAN-ORLICZ SPACES
}

\author{
AJay K. Sharma AND S.D. Sharma
}

In this paper, composition operators acting on Bergman-Orlicz spaces

$$
A_{\alpha}^{\Psi}=\left\{f \in H(\mathbb{D}):\|f\|_{A_{\alpha}^{\Psi}}=\int_{\mathbb{D}} \Psi(\log |f(z)|) d \nu_{\alpha}(z)<\infty\right\}
$$

are studied, where $\Psi$ is a non-constant, non-decreasing convex function defined on $(-\infty, \infty)$ which satisfies the growth condition $\lim _{t \rightarrow \infty} \Psi(t) / t=\infty$. In fact, under a mild condition on $\Psi$, we show that every holomorphic-self map $\varphi$ of $\mathbb{D}$ induces a bounded composition operator on $A_{\alpha}^{\Psi}$ and $C_{\varphi}$ is compact on $A_{\alpha}^{\Psi}$ if and only if it is compact on $A_{\alpha}^{2}$.

\section{INTRODUCTION}

Let $\mathbb{D}$ be the open unit disk in the complex plane $\mathbb{C}$ and $\varphi$ be a holomorphic self-map of $\mathbb{D}$. Then the equation $C_{\varphi} f=f \circ \varphi$, for $f$ analytic in $\mathbb{D}$ defines a composition operator $C_{\varphi}$ with inducing map $\varphi$. Amongst the nice composition operators on these spaces are the compact composition operators. Much of the study of compact composition operators on the spaces of analytic functions is motivated by the desire to relate the compactness of $C_{\varphi}$ with the geometric properties of $\varphi$. Commendable work in this direction was done by Schwartz [5], Shapiro and Taylor [7], MacCluer and Shapiro [4] and Shapiro [8]. The study of compact composition operators on $\mathrm{H}^{2}$ was initiated by Schwartz [5] in his thesis in 1969. Thereafter mathematicians developed elegent techniques to study composition operators on different spaces of analytic functions. MacCluer and Shapiro [4] showed that $C_{\varphi}$ is not compact whenever $\varphi$ has an angular derivative at some point of the unit circle. Non-existence of the angular derivative condition is not a sufficient condition for compactness of $C_{\varphi}$ on Hardy spaces $H^{p}$ in general. However, the angular derivative condition does characterise the compactness of $C_{\varphi}$ on $H^{p}$ if the inducing map is univalent. MacCluer developed a relationship between compact composition operators on $H^{p}$ spaces of the unit ball of $\mathbb{C}^{n}$ for $n>1$ and special class of measures on the unit disk known as Carleson measures. A connection between Carleson measure conditions for the pullback measures and composition operators on $A_{a}^{p}$ played an important role in the proof

Received 18th October, 2006

Copyright Clearance Centre, Inc. Serial-fee code: 0004-9727/07 \$A2.00+0.00. 
of MacCluer and Shapiro [4] in which they showed that the non-existence of the angular derivative condition is a necessary as well as a sufficient condition for compactness of $C_{\varphi}$ on $A_{\alpha}^{p}$. In 1987, Shapiro [8] was able to discover the connection between the essential norm of a composition operator on the Hardy space $H^{2}$ and the Nevanlinna counting function for $\varphi$, and obtained the general expression

$$
\left\|C_{\varphi}\right\|_{e}^{2}=\limsup _{|w| \rightarrow 1^{-}} \frac{N_{\varphi}(w)}{\log 1 /|w|}
$$

where by the essential norm of $C_{\varphi}$, we mean its distance, in the operator norm, from the space of compact operators on $H^{2}$. In particular, he proved that $C_{\varphi}$ is compact on $H^{2}$ if and only if $N_{\varphi}(w)=o(\log 1 /|w|)$ as $|w| \rightarrow 1$, thus providing a complete function theoretic characterisation of compact composition operators in terms of the inducing map's Nevanlinna counting function $N_{\varphi}$. Another solution to the compactness problem can be given by means of the positive measures $\lambda_{\alpha}$ that are defined on the unit circle $\partial \mathbb{D}$ by the Poisson representation

$$
\Re \frac{\alpha+\varphi(z)}{\alpha-\varphi(z)}=\int_{\partial \mathrm{D}} P(z, \zeta) d \lambda_{\alpha}(\zeta)
$$

for each $\alpha \in \partial \mathbb{D}$. These measures are often called the Aleksandrov measures of $\varphi$. In [2], Cima and Matheson showed that the essential norm of $C_{\varphi}$ on $H^{2}$ can also be expressed as

$$
\left\|C_{\varphi}\right\|_{e}^{2}=\sup _{\alpha \in \partial \mathbb{D}}\left\|\sigma_{\alpha}\right\|
$$

where $\sigma_{\alpha}$ is the singular part of $\lambda_{\alpha}$. In particular, it follows that $C_{\varphi}$ is compact on the Hardy space $H^{2}$ if and only if all the measures $\lambda_{a}$ are absolutely continuous. We are inspired by the following results.

1. If $C_{\varphi}$ is compact on one of the Hardy space $H^{p}$ for some $p(0<p<\infty)$, then it is compact on all of the Hardy spaces $H^{p}(0<p<\infty)([7])$.

2. A holomorphic composition operator is compact on $L^{1}$ if and only if it is compact on $H^{2}([6])$.

3. For an arbitrary $\varphi$ the compactness of $C_{\varphi}$ on Hardy spaces $H^{p}$ is quite different from the compactness of $C_{\varphi}$ on weighted Bergman spaces $A_{\alpha}^{p}$. MacCluer and Shapiro [4] gave a nice example of a holomorphic self-map of $\mathbb{D}$ which induces a compact composition operator on weighted Bergman space but does not induce a compact composition operator on the Hardy spaces. As a matter of fact they established the existence of an inner function $\varphi$ (holomorphic function on $\mathbb{D}$ with modulus $\leqslant 1$ everywhere on $\mathbb{D}$ and radial limit of modulus 1 at almost every point of $\partial \mathbb{D}$ ) such that $C_{\varphi}$ is compact on $A_{\alpha}^{p}$ for all $0<p<\infty$ and $\alpha>-1$. However, it is well known that no inner function can induce a compact composition operator on any of $H^{p}$ spaces. In fact, they cited the following example; 
Let

$$
\varphi(z)=\exp \int_{\partial \mathrm{D}} \frac{z+\zeta}{z-\zeta} d \mu(\zeta)
$$

where $\mu$ is a Borel measure on $\partial \mathbb{D}$ that is singular with respect to linear Lebesgue measure and

$$
\int_{\partial \mathrm{D}} \frac{d \mu(\zeta)}{|\zeta-\omega|^{2}}=\infty
$$

at every $\omega \in \partial \mathbb{D}$. Then $\varphi$ is a singular inner function which induces a compact composition operator on $A_{\alpha}^{p}$ but not on the Hardy space $H^{p}$.

Our goal in this paper is to characterise those holomorphic self-maps $\varphi$ of $\mathbb{D}$ that induce bounded and compact composition operators on the weighted Bergman-Orlicz spaces $A_{\alpha}^{\Psi}$. We shall show that like Hardy spaces and weighted Bergman spaces, every holomorphic self-map $\varphi$ of $\mathbb{D}$ induces a bounded composition operator on the weighted Bergman-Orlicz spaces $A_{\alpha}^{\Psi}$ and that $C_{\varphi}$ is compact on the Bergman-Orlicz spaces $A_{\alpha}^{\Psi}$ if and only if it is compact on any of the weighted Bergman spaces $A_{\alpha}^{p}(0<p<\infty)$.

\section{Preliminaries}

In this section we review the basic concepts and collect some essential facts that will be needed throughout the paper. Let $H(\mathbb{D})$ denote the space of all holomorphic functions in the unit disk $\mathbb{D}$ of the complex plane $\mathbb{C}$ and $d A(z)=(1 / \pi) d x d y, z=x+i y$, the normalised Lebesgue measure on $\mathbb{D}$. Let $\alpha \in(-1, \infty)$ be a real number and $\nu_{\alpha}$ be the probabilistic measure on $\mathbb{D}$ given by $d \nu_{\alpha}(z)=(\alpha+1)\left(1-|z|^{2}\right)^{\alpha} d A(z)$. For $0<p<\infty$ the weighted Bergman space $A_{\alpha}^{p}$ is defined as

$$
A_{\alpha}^{p}=\left\{f \in H(\mathbb{D}):\|f\|_{A_{\alpha}^{p}}=\left(\int_{\mathbb{D}}|f(z)|^{p} d \nu_{\alpha}(z)\right)^{1 / p}<\infty\right\} .
$$

Note that $\|f\|_{A_{\alpha}^{p}}$ is a true norm only if $1 \leqslant p<\infty$. When $0<p<1, A_{\alpha}^{p}$ is a an F-space with respect to the translation invariant metric defined by $d_{p}^{\alpha}(f, g)=\|f-g\|_{A_{\alpha}^{p}}$. The growth of functions in the Bergman space is essential in our study. The following sharp estimate tells us how fast an arbitrary function from $A_{\alpha}^{p}$ grow near the boundary. Let $f \in A_{\alpha}^{p}$. Then for every $z$ in $\mathbb{D}$, we have

$$
|f(z)| \leqslant \frac{\|f\|_{A_{\alpha}^{p}}}{\left(1-|z|^{2}\right)^{(2+\alpha) / p}}
$$

with equality if and only if $f$ is a constant multiple of the function

$$
k_{a}(z)=\left(\frac{1-|a|^{2}}{(1-\bar{a} z)^{2}}\right)^{2+\alpha / p} .
$$

It can be easily shown that $\left\|k_{a}\right\|_{A_{\alpha}^{p}} \approx 1$ with constant depending only on $\alpha$ and $p$ ([8, p. 400]). Let $\Psi$ be a non-constant, non-decreasing convex function defined on $(-\infty, \infty)$ 
which satisfy the growth condition $\lim _{t \rightarrow \infty} \Psi(t) / t=\infty$. The weighted Bergman-Orlicz space $A_{\alpha}^{\Psi}$ is defined by

$$
A_{\alpha}^{\Psi}=\left\{f \in H(\mathbb{D}):\|f\|_{A_{\alpha}^{\Psi}}=\int_{\mathbb{D}} \Psi(\log |f(z)|) d \nu_{\alpha}(z)<\infty\right\} .
$$

The Hardy-Orlicz space $H^{\Psi}$ is as usual defined by

$$
H^{\Psi}=\left\{f \in H(\mathbb{D}):\|f\|_{H^{\Psi}}=\sup _{0 \leqslant r<1} \int_{\partial \mathbb{D}} \Psi\left(\log \left|f\left(r e^{i \theta}\right)\right|\right) d \sigma(\theta)\right\},
$$

where $\sigma$ is the normalised Lebesgue measure on the unit circle $\partial \mathbb{D}$. It is clear that $H^{\Psi} \subset A_{\alpha}^{\Psi}$ for every $\Psi$ and for every $\alpha>-1$. In particular, if $\Psi(t)=\exp (p t), 0<p<\infty$ then $A_{\alpha}^{\Psi}$ is the usual weighted Bergman space $A_{\alpha}^{p}, 0<p<\infty$ with parameter $\alpha$ whereas $H^{\Psi}$ is the usual Hardy space $H^{p}, 0<p<\infty$. Throughout this paper we also assume that $\Psi$ also satisfies the following property:

$$
\Psi(2 t) \leqslant C \Psi(t) \text { for all } t>0 .
$$

We next define generalised Bergman spaces.

Let $\omega$ be a real-valued function, defined on $[0, \infty)$. If $\omega$ satisfies the following conditions.

(i) $\omega(x+y) \leqslant \omega(x)+\omega(y)$, for all $x, y \in[0, \infty)$;

(ii) $\omega$ is continuous at zero, from the right;

(iii) $\omega(x)=0$ if and only if $x=0$;

we say that $\omega$ is a modulus function. Some examples of modulus function are $x^{p}, 0$ $<p \leqslant 1$ and $\log (1+x)$. In fact, if $\omega$ is a modulus function, then so is $\omega /(1+\omega)$. Let $\omega$ be a modulus function. The generalised weighted Bergman space $A_{\alpha}^{\omega}$ is defined to be the collection of all analytic functions $f$ on $\mathbb{D}$ for which

$$
\|f\|_{A_{\alpha}}=\int_{\mathbb{D}} \omega(|f(z)|) d \nu_{\alpha}(z)<\infty .
$$

The spaces $A_{\alpha}^{\omega}$ is not necessarily complete. However, if $\omega(|f|)$ is subharmonic in $\mathbb{D}$ for every $f \in H(\mathbb{D})$, then $A_{\alpha}^{\omega}$ becomes an F-space. In view of Hasumi and Kataoka [3, Lemma 5.1], the above condition on $\omega$ is equivalent the following, ' $\omega\left(e^{t}\right)$ is a convex function'. Throughout this paper we shall assume that our modulus function also satisfy the following properties.

(i) $\omega(|f|)$ is a subharmonic function for every $f \in H(\mathbb{D})$

(ii) $\omega$ is strictly increasing;

(iii) $\omega(x y) \leqslant \omega(x)+\omega(y)$ for all $x, y \in[0, \infty)$; 
$2.1 \alpha$-Carleson measure. For $\zeta \in \partial \mathbb{D}$ and $0<\delta<2$, let

$$
S(\delta, \zeta)=\{z \in \mathbb{D}:|z-\zeta|<\delta\}
$$

A positive Borel measure $\mu$ on $\mathbb{D}$ is called $\alpha$-Carleson measure if

$$
\sup _{\delta>0} \sup _{\zeta \in \partial \mathbb{D}} \frac{\mu(S(\delta, \zeta))}{\delta^{\alpha+2}}<\infty
$$

and it will be called a vanishing Carleson measure if

$$
\lim _{\delta \rightarrow 0} \sup _{\zeta \in \partial \mathbb{D}} \frac{\mu(S(\delta, \zeta))}{\delta^{\alpha+2}}=0 .
$$

We use the sets $S(\delta, \zeta)$ as the Carleson sets along with a more convenient choice of pseudohyperbolic disks. We now incorporate a few lines from Axler's paper [1]. For any fixed $a \in \mathbb{D}$, let $\tau_{a}$ be the function defined by

$$
\tau_{a}(z)=\frac{a-z}{1-\bar{a} z}
$$

for $z \in \mathbb{D}$. The function $\tau_{a}$ is an automorphism of $\mathbb{D}$. For $a$ and $z$ in $\mathbb{D}$, the pseudohyperbolic distance $d$ between $a$ and $z$ is defined by

$$
d(a, z)=\left|\tau_{a}(z)\right|
$$

For $0<r<1$ and $a \in \mathbb{D}$, denote by $D(a, r)$, the disk whose pseudohyperbolic centre is $a$ and whose pseudohyperbolic radius is $r$ :

$$
D(a, r)=\{z \in \mathbb{D}: d(a, z)<r\}
$$

Since $\tau_{a}$ is a linear fractional tansformation, the pseudohyperbolic disk $D(a, r)$ is also a Euclidean disk. The Euclidean centre and Euclidean radius of $D(a, r)$ are

$$
\frac{1-r^{2}}{1-r^{2}|a|^{2}} a \text { and } \quad \frac{1-|a|^{2}}{1-r^{2}|a|^{2}} r
$$

respectively. The notation $|D(a, r)|_{A}$ will denote the area of $D(a, r)$. For fixed $0<r<1$ the area of $D(a, r)$ has the estimation:

$$
|D(a, r)|_{A} \approx\left(1-|a|^{2}\right)^{2} \approx\left(1-|z|^{2}\right)^{2} \approx|D(z, r)|_{A}
$$

for $z \in D(a, r)$, where $\approx$ means that the two quantities are bounded above and below by the constants independent of $a$. For fixed $0<r<1$, it is also known that for $z \in D(a, r)$,

$$
|1-\bar{a} z| \approx\left(1-|a|^{2}\right)
$$


Also for each $D(a, r)$, there is a $\zeta \in \partial \mathbb{D}$ so that $D(a, r) \subset S(\delta, \zeta)$ for $\delta \approx 1-|a|$ and for fixed $r, 0<r<1$

$$
(\alpha+1) \int_{D(a, r)}\left(1-|z|^{2}\right)^{\alpha} d A(z) \approx\left(1-|a|^{2}\right)^{\alpha+2}
$$

The following Lemma was proved by Axler in [1].

LEMmA 2.2. Let $0<r<1$. Then there is a sequence $\left\{a_{n}\right\}$ in $\mathbb{D}$ and a positive integer $M$ such that $\cup_{n=1}^{\infty} D\left(a_{n}, r\right)=\mathbb{D}$ and each $z \in \mathbb{D}$ is in at most $M$ of the pseudohyperbolic disks

$$
D\left(a_{1}, \frac{1+r}{2}\right), D\left(a_{2}, \frac{1+r}{2}\right), D\left(a_{3}, \frac{1+r}{2}\right), \ldots
$$

2.3. ANGUlar DeRIVATIVE. ([9, p. 56]) We say that $\varphi$ has a finite angular derivative at a point $\zeta \in \partial \mathbb{D}$ if there is a point $\omega \in \partial \mathbb{D}$ such that the difference quotient

$$
\frac{\varphi(z)-\omega}{z-\zeta}
$$

has a finite limit, as $z$ tends non-tangentially to $\zeta$.

The connection between composition operators and angular derivative is made by the following classical theorem.

2.4. Julia-Caratheodory Theorem. ( $(9$, p. 57$]$.) For $\zeta \in \partial \mathbb{D}$, the following are equivalent:

(1) $\varphi$ has an angular derivative at $\zeta$.

(2) $\varphi$ has a non-tangential limit of modulus 1 at $\zeta$, and the complex derivative $\varphi^{\prime}$ has a finite limit at $\zeta$. In this case, the limit of $\varphi^{\prime}$ is $\varphi^{\prime}(\zeta)$.

(3) $\liminf _{z \rightarrow \zeta}(1-|\varphi(z)|) /(1-|z|)=d<\infty$.

(For more information on the Julia-Caratheodory Theorem and its connection with composition operators see [4, Section 3], or [9, Chapter 4].)

The next criteria of compactness of $C_{\varphi}$, is due to MacCluer and Shapiro [4] and is useful in the main result of this paper.

THEOREM 2.5. Let $\varphi$ be a holomorphic self-map of $\mathbb{D}$. Then the following conditions are equivalent:

(1) $C_{\varphi}$ is compact on $A_{\alpha}^{2}$.

(2) $\varphi$ has an angular derivative at no point on the boundary $\partial \mathbb{D}$ of $\mathbb{D}$.

(3) The pull back measure $\nu_{\alpha} \circ \varphi^{-1}$ is a vanishing Carleson measure on $\mathbb{D}$.

Lemma 2.6. For a fixed $r, 0<r<1$, there exists a positive constant $C$ depending upon $r$ such that

$$
\Psi(\log |f(w)|) \leqslant \frac{C}{\left(1-|w|^{2}\right)^{2+\alpha}} \int_{D(w, r)} \Psi(\log |f(z)|) d \nu_{\alpha}(z)
$$


for $f$ analytic in $\mathbb{D}$, and $z \in \mathbb{D}$.

ProOF: Since $D(0, r)$ is a Euclidean disk with centre 0 and radius $r$ and $\Psi(\log |f(z)|)$ is subharmonic for all analytic $f$, we have

$$
\begin{aligned}
\Psi(\log |f(0)|) & \leqslant \frac{1}{|D(0, r)|_{A}} \int_{D(0, r)} \Psi(\log |f(z)|) d A(z) \\
& \leqslant \frac{1}{r^{2}} \int_{D(0, r)} \Psi(\log |f(z)|) d A(z) .
\end{aligned}
$$

Replacing $f$ by $f \circ \tau_{w}$ and changing variable, we obtain

$$
\Psi(\log |f(w)|) \leqslant \frac{1}{|D(w, r)|_{A}} \int_{D(w, r)} \Psi(\log |f(z)|) \frac{\left(1-|z|^{2}\right)^{2}}{|1-\bar{z} w|^{4}} d A(z) .
$$

Since $0<r<1,(2.1)$ and (2.2) imply that there is a constant $C>0$, depending upon $r$, such that

$$
\Psi(\log |f(w)|) \leqslant \frac{C}{\left(1-|w|^{2}\right)^{2+\alpha}} \int_{D(w, r)} \Psi(\log |f(z)|) \nu_{\alpha}(z) .
$$

The following lemma asserts that sequences that are norm bounded in $A_{\alpha}^{\Psi}$ are uniformly bounded on compact subsets of $\mathbb{D}$. In other words, for $0<r<1$ there has to be a uniform bound for all point-evaluations corresponding to points in $r \mathbb{D}$.

Lemma 2.7. For $z \in \mathbb{D}$,

$$
|f(z)| \leqslant \exp \left(\Psi^{-1}\left(\frac{C\|f\|_{A_{\alpha}^{\psi}}}{\left(1-|z|^{2}\right)^{\alpha+2}}\right)\right)
$$

for all $f \in A_{\alpha}^{\Psi}$.

Proof is an easy consequence of Lemma 2.6 .

\section{Boundedness of $C_{\varphi}$ ON $A_{\alpha}^{\Psi}$}

In this section we show that every holomorphic self-map $\varphi$ of $\mathbb{D}$ induces a bounded composition operator on Bergman-Orlicz spaces $A_{\alpha}^{\Psi}$.

We need the following lemma.

LEMMA 3.1. If $\mu$ is an $\alpha$-Carleson measure on $\mathbb{D}$, then there is a constant $C$ such that

$$
\int_{\mathbb{D}} \Psi(\log (|f(w)|)) d \mu(w) \leqslant C \int_{\mathbf{D}} \Psi(\log (|f(w)|)) d \nu_{\boldsymbol{\alpha}}(w)
$$

for any $f \in A_{\alpha}^{\Psi}$.

Proof: Fix $0<r<1$. Pick a sequence $\left\{a_{n}\right\}$ in $\mathbb{D}$ satisfying the conditions of the 
Lemma 2.2. For $f \in A_{\alpha}^{\Psi}$, we have

$$
\begin{aligned}
\int_{\mathbb{D}} \Psi(\log (|f(w)|)) & d \mu(w) \\
& \leqslant \sum_{n=1}^{\infty} \int_{D\left(a_{n}, r\right)} \Psi(\log (|f(w)|)) d \mu(w) \\
& \leqslant \sum_{n=1}^{\infty} \mu\left(D\left(a_{n}, r\right)\right) \sup \left\{\Psi(\log (|f(w)|)): w \in D\left(a_{n}, r\right)\right\} \\
& \leqslant C^{\prime} \sum_{n=1}^{\infty} \frac{\mu\left(D\left(a_{n}, r\right)\right)}{\nu_{\alpha}\left(D\left(a_{n}, r\right)\right)} \int_{D\left(a_{n},(1+r) / 2\right)} \Psi(\log (|f(w)|)) d \nu_{\alpha}(w) \\
& \leqslant C^{\prime} C^{\prime \prime} \sum_{n=1}^{\infty} \frac{\mu\left(S\left(1-\left|a_{n}\right|, \zeta\right)\right)}{\left(1-\left|a_{n}\right|^{2}\right)^{a+2}} \int_{D\left(a_{n},(1+r) / 2\right)} \Psi(\log (|f(w)|)) d \nu_{\alpha}(w) \\
& \leqslant C^{\prime} C^{\prime \prime} C^{\prime \prime \prime} \sum_{n=1}^{\infty} \int_{D\left(a_{n},(1+r) / 2\right)} \Psi(\log (|f(w)|)) d \nu_{\alpha}(w) \\
& \leqslant C M \int_{\mathbb{D}} \Psi(\log (|f(w)|)) d \nu_{\alpha}(w), \quad \text { where } C=C^{\prime} C^{\prime \prime} C^{\prime \prime \prime} .
\end{aligned}
$$

The following theorem provides a necessary and sufficient condition for boundedness of $C_{\varphi}: A_{\alpha}^{\Psi} \rightarrow A_{\alpha}^{\Psi}$ in terms of Carleson measure condition for the pull-back measure $\nu_{\alpha} \circ \varphi^{-1}$ on $\mathbb{D}$.

THEOREM 3.2. For a holomorphic self-map $\varphi$ of $\mathbb{D}, C_{\varphi}: A_{\alpha}^{\Psi} \rightarrow A_{\alpha}^{\Psi}$ is bounded if and only if the pull-back measure $\mu_{\varphi, \alpha}=\nu_{\alpha} \circ \varphi^{-1}$ is an $\alpha$-Carleson measure on $\mathbb{D}$.

Proof: Suppose $C_{\varphi}$ is bounded. We need to verify that the pull-back measure $\mu_{\varphi, \alpha}=\nu_{\alpha} \circ \varphi^{-1}$ is a $\alpha$-Carleson measure on $\mathbb{D}$. Assume $0<\delta<1$ and $\zeta \in \partial \mathbb{D}$. Let $a=(1-\delta) \zeta$ and consider the function

$$
g_{a}\left(e^{i \theta}\right)=\frac{\left(1-|a|^{2}\right)^{\alpha+2}}{\left(\left|1-\bar{a} e^{i \theta}\right|^{2}\right)^{2(\alpha+2)}} .
$$

Then $g_{a}$ is non-negative and $g_{a} \in L^{1}(d \sigma)$. Let $K\left(e^{i \theta}\right)=(\Psi)^{-1}\left(g_{a}\left(e^{i \theta}\right)\right)$. Then $K$ is well defined, for $\Psi$ is strictly increasing in the range of $g_{a}$. Since $\Psi$ is convex, $\Psi^{-1}$ is concave and so there is a constant $C>0$ such that $\Psi^{-1}(x) \leqslant C x$ for sufficiently large $x$. Thus $K \in L^{1}(d \sigma)$. We set

$$
h(z)=\exp \left\{\int_{0}^{2 \pi} H\left(z, e^{i t}\right) K\left(e^{i t}\right) d \sigma(t)\right\},
$$

where $H\left(z, e^{i t}\right)$ denotes the Herglotz kernel for $\mathbb{D}$; namely, $H\left(z, e^{i t}\right)=\left(e^{i t}+z\right) /\left(e^{i t}-z\right)$, $z \in \mathbb{D}$. Then

$$
\Psi\left(\log \left|h\left(e^{i \theta}\right)\right|\right)=\Psi\left(\log \left(\exp \left(\Psi^{-1} g_{a}\left(\left(e^{i \theta}\right)\right)\right)\right)\right)=g_{a}\left(e^{i \theta}\right) \in L^{1}(d \sigma) .
$$


This means that $h \in H^{\Psi}$ and so also in $A_{\boldsymbol{\alpha}}^{\Psi}$.

$$
\begin{aligned}
\|h\|_{A_{\alpha}^{\Psi}} & =\int_{\mathbb{D}} \Psi(\log |h(z)|) d \nu_{\alpha}(z) \\
& =\int_{\mathbb{D}} \Psi(\log (\exp (K(z)))) d \nu_{\alpha}(z) \\
& \leqslant \int_{\mathbb{D}} \frac{\left(1-|a|^{2}\right)^{(\alpha+2)}}{|1-\bar{a} z|^{2(\alpha+2)}} d \nu_{\alpha}(z) \\
& \approx 1
\end{aligned}
$$

Since $C_{\varphi}$ is bounded, there is a constant $C$ such that

$$
\left\|C_{\varphi} h\right\|_{A_{\alpha}^{\Psi}} \leqslant C\|h\|_{A_{\alpha}^{\Psi}} \leqslant C .
$$

That is

$$
\begin{aligned}
C & \geqslant \int_{\mathbb{D}} \Psi(\log |(h \circ \varphi)(z)|) d \nu_{\alpha}(z) \\
& =\int_{\mathbb{D}} \Psi\left(\log \mid(h(z) \mid) d \nu_{\alpha} \varphi^{-1}(z)\right. \\
& \geqslant \int_{\mathbb{D}} \Psi(\log (\exp (K(z)))) d \nu_{\alpha} \varphi^{-1}(z) \\
& \geqslant \int_{\mathbb{D}} \Psi(K(z)) d \nu_{\alpha} \varphi^{-1}(z) \\
& =\int_{\mathbb{D}} \frac{\left(1-|a|^{2}\right)^{(\alpha+2)}}{|1-\bar{a} z|^{2(\alpha+2)}} d \mu_{\varphi, \alpha}(z) .
\end{aligned}
$$

On the other hand

$$
\begin{aligned}
\frac{\left(1-|a|^{2}\right)^{(\alpha+2)}}{|1-\bar{a} z|^{2(\alpha+2)}} & \geqslant \Re\left(\frac{\left(1-|a|^{2}\right)^{(\alpha+2)}}{(1-\bar{a} z)^{2(\alpha+2)}}\right) \\
& =\frac{\left(1-|a|^{2}\right)^{(\alpha+2)}}{(1-|a|)^{2(\alpha+2)}} \Re\left(\frac{1-|a|}{1-\bar{a} z}\right)^{2(\alpha+2)} \\
& =\frac{\left(1-|a|^{2}\right)^{(\alpha+2)}}{(1-|a|)^{2(\alpha+2)}} \Re\left(1+\frac{|a|(1-z \bar{\zeta})}{(1-|a|)}\right)^{-2(\alpha+2)},\left(\zeta=\frac{a}{|a|}\right) \\
& >\frac{\left(1-|a|^{2}\right)^{(\alpha+2)}}{(1-|a|)^{2(\alpha+2)}} \frac{1}{2^{\alpha+2}} \\
& \geqslant \frac{1}{(2 \delta)^{\alpha+2}}
\end{aligned}
$$

if $(|1-z \bar{\zeta}|) /(1-|a|)<\gamma_{0}$ for some fixed $\gamma_{0}>0$; that is, if $z \in S\left(\gamma_{0} \delta, \zeta\right)$. Hence for all 
$\zeta \in \partial \mathbb{D}$ and $0<\delta<1$, we have

$$
\begin{aligned}
C & \geqslant \int_{\mathbb{D}} \frac{\left(1-|a|^{2}\right)^{\alpha+2}}{|1-\bar{a} z|^{2(\alpha+2)}} d \mu_{\varphi, \alpha}(z) \\
& \geqslant \int_{S\left(\gamma_{0} \delta, \zeta\right)} \frac{1}{(2 \delta)^{\alpha+2}} d \mu_{\varphi, \alpha}(z) \\
& \geqslant \frac{1}{(2 \delta)^{\alpha+2}} \mu_{\varphi, \alpha}\left(S\left(\gamma_{0} \delta, \zeta\right)\right),
\end{aligned}
$$

that is, $\nu_{\alpha} \varphi^{-1}\left(S\left(\gamma_{0} \delta, \zeta\right)\right) \leqslant C \delta^{\alpha+2}$ and hence $\nu_{\alpha} \varphi^{-1}$ is an $\alpha$-Carleson measure on $\mathbb{D}$. Conversely, suppose $\nu_{\alpha} o \varphi^{-1}$ is an $\alpha$-Carleson measure. Then by Lemma 3.1, we have for $f \in A_{\alpha}^{\Psi}$,

$$
\begin{aligned}
\left\|C_{\varphi} f\right\|_{A_{\boldsymbol{\alpha}}^{\Psi}} & =\int_{\mathbb{D}} \Psi(\log (|(f \circ \varphi)(w)|)) d \nu_{\alpha}(w) \\
& =\int_{\mathbb{D}} \Psi(\log (|f(w)|)) d \mu_{\varphi, \boldsymbol{\alpha}}(w) \\
& \leqslant C \int_{\mathbb{D}} \Psi(\log |f(w)|) d \nu_{\alpha}(w) \\
& =C\|f\|_{A_{\alpha}^{\Psi} .}
\end{aligned}
$$

This completes the proof.

Remark. In [4, Theorem 4.3] MacCluer and Shapiro assert that $C_{\varphi}$ is bounded on $A_{\alpha}^{p}$ if and only if $\mu_{\varphi, \alpha}=\nu_{\alpha} \circ \varphi^{-1}$ is an $\alpha$-Carleson measure on $\mathbb{D}$. Thus by Theorem 3.1 above $C_{\varphi}$ is bounded on $A_{\alpha}^{\Psi}$ if and only if it is bounded on $A_{\alpha}^{p}$. But by [4, Theorem 3.4] of MacCluer and Shapiro, every holomorphic self-map $\varphi$ of $\mathbb{D}$ induces a bounded composition operator on $A_{\alpha}^{p}$ for all $0<p<\infty$ and $\alpha>-1$. Hence every holomorphic self-map $\varphi$ of $\mathbb{D}$ induces a bounded composition operator on $A_{\alpha}^{\psi}$ also.

\section{Compactness of $C_{\varphi}$ on $A_{\alpha}^{\Psi}$}

The following lemma characterises the compactness of $C_{\varphi}$ on $A_{\alpha}^{\Psi}$ in terms of sequential convergence. It can be proved on similar lines as in [6, Proposition 3.11]. So we omit the details.

LEMMA 4.1. Let $\varphi$ be holomorphic self-map of $\mathbb{D}$. Then $C_{\varphi}$ is compact on $A_{\alpha}^{\Psi}$ if and only if for every sequence $\left\{f_{n}\right\}$ which is norm bounded and converges to zero uniformly on compact subsets of $\mathbb{D}$, we have $\left\|f_{n} \circ \varphi\right\|_{A_{a}^{\Psi}} \rightarrow 0$.

We now characterise compact composition operators on $A_{\alpha}^{\Psi}$.

THEOREM 4.2. Let $\varphi$ be a holomorphic self-map of $\mathbb{D}$. Then the following are equivalent:

(1) $C_{\varphi}$ is compact on $A_{\alpha}^{\Psi}$.

(2) The pull-back measure $\mu_{\varphi, \alpha}=\nu_{\alpha} \circ \varphi^{-1}$ is a vanishing Carleson measure on $\mathbb{D}$. 
(3) $C_{\varphi}$ is compact on $A_{\alpha}^{2}$.

(4) $\varphi$ has an angular derivative at no point on the boundary $\partial \mathbb{D}$ of $\mathbb{D}$.

PROoF: In view of Theorem 2.5 , we need only to show that $(1) \Rightarrow(2)$ and $(4) \Rightarrow(1)$ To prove (1) $\Rightarrow(2)$, let us suppose that $C_{\varphi}$ is compact. Assume $0<\delta<1 / 2$ and $\zeta \in \partial \mathbb{D}$. Let $a=(1-\delta) \zeta$. Consider the family of functions

$$
f_{a}(z)=2 \frac{(1-|a|)}{(1-\bar{a} z)} h(z)
$$

where $h$ is the same function as in Theorem 3.2. Then

$$
\begin{aligned}
\left\|f_{a}\right\|_{A_{\alpha}^{\Psi}} & =\int_{\mathbb{D}} \Psi\left(\log \left|f_{a}(z)\right|\right) d \nu_{\alpha}(z) \\
& \leqslant \int_{\mathbb{D}} \Psi\left(\log \left(2\left|h_{a}(z)\right|\right)\right) d \nu_{\alpha}(z) \\
& =\int_{\mathbb{D}} \Psi\left(\log 2+\log (\exp (K(z) \mid)) d \nu_{\alpha}(z)\right. \\
& \leqslant \frac{1}{2} \Psi(2 \log 2)+\frac{1}{2} C \int_{\mathbb{D}} \frac{\left(1-|a|^{2}\right)^{(\alpha+2)}}{|1-\bar{a} z|^{2(\alpha+2)}} d \nu_{\alpha}(z) \\
& \leqslant \frac{1}{2} \Psi(2 \log 2)+\frac{1}{2} C .
\end{aligned}
$$

Clearly $f_{a} \rightarrow 0$ uniformly on compact subsets of $\mathbb{D}$ as $|a| \rightarrow 1$. Again, as in the proof of Theorem 3.2, there exist $\gamma_{0}>0$ such that if $z \in S\left(\gamma_{0} \delta, \zeta\right)$, then

$$
\frac{\left(1-|a|^{2}\right)^{(\alpha+2)}}{|1-\bar{a} z|^{2(a+2)}} \geqslant \frac{1}{(2 \delta)^{\alpha+2}} \text {. }
$$

Hence for $z \in S\left(\gamma_{0} \delta, \zeta\right)$,

$$
\begin{aligned}
\Psi\left(\log \left|f_{a}(z)\right|\right) & =\Psi\left(\log 2 \frac{(1-|a|)}{(1-\bar{a} z)} h(z)\right) \\
& \geqslant \Psi(\log (\exp (K(z)))) \\
& \geqslant \Psi\left(\log \left(\exp \Psi^{-1}\left(\frac{1}{(2 \delta)^{\alpha+2}}\right)\right)\right) \\
& =\frac{1}{(2 \delta)^{\alpha+2}} .
\end{aligned}
$$

Therefore, we have for any $\zeta \in \partial \mathbb{D}$ and $0<\delta<1 / 2$

$$
\begin{aligned}
\frac{1}{(2 \delta)^{\alpha+2}} \mu_{\varphi, \alpha}\left(S\left(\gamma_{0} \delta, \zeta\right)\right) & \leqslant \int_{S\left(\gamma_{0} \delta, \zeta\right)} \Psi\left(\log \left|f_{a}(z)\right|\right) d \mu_{\varphi, \alpha}(z) \\
& \leqslant \int_{\mathbf{D}} \Psi\left(\log \left|\left(f_{a} \circ \varphi\right)(z)\right|\right) d \nu_{\alpha}(z) \\
& =\left\|C_{\varphi} f_{a}\right\|_{A_{\alpha}^{\Psi} .}
\end{aligned}
$$


But compactness of $C_{\varphi}$ forces $\left\|C_{\varphi} f_{a}\right\|_{A_{a}^{\varphi}}$ to tend to zero as $\delta \rightarrow 0$, which implies that

$$
\lim _{\delta \rightarrow 0} \frac{1}{(2 \delta)^{\alpha+2}} \mu_{\varphi, \alpha}\left(S\left(\gamma_{0} \delta, \zeta\right)\right)=0
$$

uniformly in $\zeta \in \partial D$. Hence $\mu_{\varphi, \alpha}=\nu_{\alpha} \circ \varphi^{-1}$ is a vanishing Carleson measure on $\mathbb{D}$.

(4) $\Rightarrow$ (1) Suppose that $\nu_{\alpha} \varphi^{-1}$ is a vanishing Carleson measure on $\mathbb{D}$. Then

$$
\frac{\mu_{\varphi, \alpha}\left(S\left(\gamma_{0} \delta, \zeta\right)\right.}{\delta^{\alpha+2}} \rightarrow 0 \text { uniformly in } \zeta \text { as } \delta \rightarrow 0
$$

that is

$$
\frac{\mu_{\varphi, \alpha}(D(w, r))}{(1-|w|)^{\alpha+2}} \rightarrow 0 \text { uniformly in } w \text { as }|w| \rightarrow 1 .
$$

Fix any $r>0$ and a sequence $\left\{w_{n}\right\}$ as in Lemma 2.2. Since $\left|w_{n}\right| \rightarrow 1$ as $n \rightarrow \infty$,

$$
\lim _{n \rightarrow \infty} \frac{\mu_{\varphi, \alpha}\left(D\left(w_{n}, r\right)\right)}{\left(1-\left|w_{n}\right|\right)^{\alpha+2}}=0
$$

Thus, for every $\varepsilon>0$, we can find a positive integer $N_{0}$ such that

$$
\frac{\mu_{\varphi, \alpha}\left(D\left(w_{n}, r\right)\right)}{\left(1-\left|w_{n}\right|\right)^{\alpha+2}}<\varepsilon, \quad n \geqslant N_{0}
$$

Fix a sequence $\left\{f_{m}\right\}$ that is bounded by a finite constant $M$ in $A_{\alpha}^{\Psi}$ and converges to zere uniformly on compact subsets of $\mathbb{D}$. By Lemma 4.1, it is enough to show that $\left\|f_{m} \circ \varphi\right\|_{A_{\alpha}^{\varpi}} \rightarrow 0$. Now

$$
\begin{aligned}
\left\|C_{\varphi} f_{m}\right\|_{A_{\alpha}^{\Psi}} & =\int_{\mathbb{D}} \Psi\left(\log \left(\left|\left(f_{m} \circ \varphi\right)(z)\right|\right)\right) d \nu_{\alpha}(z) \\
& \leqslant \sum_{n=1}^{\infty} \int_{D\left(w_{n}, r\right)} \Psi\left(\log \left(\left|\left(f_{m}(z)\right)\right|\right)\right) d \mu_{\varphi, \alpha}(z) \\
& =\sum_{n=1}^{N_{0}} \int_{D\left(w_{n}, r\right)} \Psi\left(\log \left(\left|\left(f_{m}(z)\right)\right|\right)\right) d \mu_{\varphi, \alpha}(z) \\
& \quad+\sum_{n=N_{0}}^{\infty} \int_{D\left(w_{n}, r\right)} \Psi\left(\log \left(\mid\left(f_{m}(z) \mid\right)\right) d \mu_{\varphi, \alpha}(z)\right.
\end{aligned}
$$

Again as in the proof of the Lemma 3.1, there is a constant $C>0$ such that

$$
\begin{aligned}
\sum_{n=N_{0}}^{\infty} \int_{D\left(w_{n}, r\right)} \Psi\left(\log \left(\mid\left(f_{m}(z) \mid\right)\right) d \mu_{\varphi, \alpha}(z)\right) \\
\leqslant C \sum_{n=N_{0}}^{\infty} \frac{\mu_{\alpha} \varphi^{-1}\left(D \left(w_{n}, r\right.\right.}{\left(1-\left|w_{n}\right|\right)^{\alpha+2}} \int_{D\left(w_{n},(1+r) / 2\right)} \Psi\left(\log \left(\left|f_{m}(z)\right|\right)\right) d \mu_{\varphi, \alpha}(z) \\
\leqslant \varepsilon C \int_{D\left(w_{n},(1+r) / 2\right)} \Psi\left(\log \left(\left|f_{m}(z)\right|\right)\right) d \mu_{\varphi, \alpha}(z) \\
=\varepsilon C M \int_{\mathbb{D}} \Psi\left(\log \left(\left|f_{m}(z)\right|\right)\right) d \mu_{\varphi, \alpha} \\
=\varepsilon C M N^{p}
\end{aligned}
$$


for all $m \geqslant 1$. Since $\left\{f_{m}\right\}$ converges to zero on each compact subset of $\mathbb{D}$, we have

$$
\lim _{m \rightarrow \infty} \sum_{n=1}^{N_{0}} \int_{D\left(w_{n}, r\right)} \Psi\left(\log \left(\left|f_{m}(z)\right|\right)\right) d \mu_{\varphi, \alpha}(z)=0 .
$$

Since $\varepsilon>0$ was arbitrary, we see that $\left\|C_{\varphi} f_{m}\right\|_{A_{\alpha}^{\psi}} \rightarrow 0$. Hence $C_{\varphi}$ is compact.

We are now going to see that our result also holds for generalised Bergman spaces $A_{\alpha}^{\omega}$. Recently, Stevic in [10] have studied composition operators on generalised Bergman spaces. He proved that like other spaces of analytic functions of the open unit disk, every analytic self-map of $\mathbb{D}$ induces a bounded composition operator. He also provided several necessary and sufficient conditions for $C_{\varphi}$ to be compact.

The following lemma characterises the compactness of $C_{\varphi}$ on $A_{\alpha}^{\omega}$ in terms of sequential convergence (see [10]).

THEOREM 4.3. Let $\varphi$ be holomorphic self-map of $\mathbb{D}$. Then $C_{\varphi}$ is compact on $A_{\alpha}^{\omega}$ if and only if for every sequence $\left\{f_{n}\right\}$ which is norm bounded and converges to zero uniformly on compact subsets of $\mathbb{D}$, we have $\left\|f_{n} \circ \varphi\right\|_{A_{\alpha}^{\omega}} \rightarrow 0$.

We now characterise compact composition operators on $A_{\alpha}^{\omega}$.

THEOREM 4.4. Let $\varphi$ be a holomorphic self-map of $\mathbb{D}$. Then the following are equivalent:

(1) $C_{\varphi}$ is compact on $A_{\alpha}^{\omega}$.

(2) The pull back measure $\nu_{\alpha} \circ \varphi^{-1}$ is a vanishing Carleson measure on $\mathbb{D}$.

(3) $C_{\varphi}$ is compact on $A_{\alpha}^{2}$.

(4) $\varphi$ has angular derivative at no point on the boundary $\partial \mathbb{D}$ of $\mathbb{D}$.

Proof: In view of Theorem 2.5, we need only to show that (1) $\Rightarrow(2)$ and (4) $\Rightarrow$ (1) To prove (1) $\Rightarrow(2)$, suppose that $C_{\varphi}$ is compact. Assume $0<\delta<1 / 2$ and $\zeta \in \partial D$. Let $a=(1-\delta) \zeta$, and consider the function

$$
g_{a}\left(e^{i \theta}\right)=\frac{\left(1-|a|^{2}\right)^{\alpha+2}}{\left(\left|1-\bar{a} e^{i \theta}\right|^{2}\right)^{2(\alpha+2)}} .
$$

Then $g_{a}$ is non-negative and $g_{a} \in L^{1}(d \sigma)$. Let $K(x)=\omega\left(e^{x}\right)$. By Hasumi and Kataoka in [3, Lemma 5.1], $K$ is convex. Let $\Omega\left(e^{i \theta}\right)=(K)^{-1}\left(g_{a}\left(e^{i \theta}\right)\right)$. Then $\Omega$ is well defined, for $K$ is strictly increasing in the range of $g_{a}$. Since $K$ is convex, $K^{-1}$ is concave and so there is a constant $C>0$ such that $K^{-1}(x) \leqslant C x$ for sufficiently large $x$. Thus $\Omega \in L^{1}(d \sigma)$. We set

$$
h(z)=\exp \left\{\int_{0}^{2 \pi} H\left(z, e^{i t}\right) \Omega\left(e^{i t}\right) d \sigma(t)\right\}
$$

where $H\left(z, e^{i t}\right)$ denotes the Herglotz kernel for $\mathbb{D}$; namely, $H\left(z, e^{i t}\right)=\left(e^{i t}+z\right) /\left(e^{i t}-z\right)$, $z \in \mathbb{D}$. Then

$$
\omega\left(\left|h\left(e^{i \theta}\right)\right|\right)=\omega\left(\exp \left(\Omega\left(e^{i \theta}\right)\right)\right)=g_{a}\left(e^{i \theta}\right) \in L^{1}(d \sigma) .
$$


This means that $h \in H^{\omega}$ and so also in $A_{\alpha}^{\omega}$. Consider the family of functions

$$
f_{a}(z)=2 \frac{(1-|a|)}{(1-\bar{a} z)} h(z),
$$

Then

$$
\begin{aligned}
\left\|f_{a}\right\|_{A_{\alpha}^{\omega}} & =\int_{\mathbb{D}} \omega\left(\left|f_{a}(z)\right|\right) d \nu_{\alpha}(z) \\
& =\int_{\mathbb{D}} \omega\left(2 \frac{1-|a|^{2}}{|1-\bar{a} z|}\left|h_{a}(z)\right|\right) d \nu_{\alpha}(z) \\
& =\int_{\mathbb{D}} \omega(2 \exp (\Omega(z))) d \nu_{\alpha}(z) \\
& \leqslant \omega(2)+\int_{\mathbb{D}} \omega(\exp (\Omega(z))) d \nu_{\alpha}(z) \\
& \leqslant \omega(2)+\int_{\mathbb{D}} \frac{\left(1-|a|^{2}\right)^{(\alpha+2)}}{|1-\bar{a} z|^{2(\alpha+2)}} d \nu_{\alpha}(z) \\
& \leqslant \omega(2)+1
\end{aligned}
$$

Clearly $f_{a} \rightarrow 0$ uniformly on compact subsets of $\mathbb{D}$ as $|a| \rightarrow 1$. Again, as in the proof of Theorem 3.2, there exist $\gamma_{0}>0$ such that if $z \in S\left(\gamma_{0} \delta, \zeta\right)$, then

$$
\frac{\left(1-|a|^{2}\right)^{(\alpha+2)}}{|1-\bar{a} z|^{2(\alpha+2)}} \geqslant \frac{1}{(2 \delta)^{\alpha+2}} \text {. }
$$

Hence for $z \in S\left(\gamma_{0} \delta, \zeta\right)$,

$$
\begin{aligned}
\Omega\left(\log \left|f_{a}(z)\right|\right) & =\omega\left(2 \frac{(1-|a|)}{(1-\bar{a} z)} h(z)\right) \\
& \geqslant \omega((\exp (\Omega(z))) \\
& \geqslant \omega\left(\exp K^{-1}\left(\frac{1}{(2 \delta)^{\alpha+2}}\right)\right) \\
& =\frac{1}{(2 \delta)^{\alpha+2}} .
\end{aligned}
$$

Therefore, we have for any $\zeta \in \partial D$ and $0<\delta<1$

$$
\begin{aligned}
\frac{1}{(2 \delta)^{\alpha+2}} \nu_{\alpha} \varphi^{-1}\left(S\left(\gamma_{0} \delta, \zeta\right)\right) & \leqslant \int_{S\left(\gamma_{0} \delta, \zeta\right)} \omega\left(\left|f_{a}(z)\right|\right) d \nu_{\alpha} \varphi^{-1}(z) \\
& \leqslant \int_{\mathbf{D}} \omega\left(\left|\left(f_{a} \circ \varphi\right)(z)\right|\right) d \nu_{\alpha}(z) \\
& =\left\|C_{\varphi} f_{a}\right\|_{A_{\alpha}^{\omega}} .
\end{aligned}
$$

But compactness of $C_{\varphi}$ forces $\left\|C_{\varphi} f_{a}\right\|_{A_{a}^{\omega}}$ to tend to zero as $\delta \rightarrow 0$, which implies that

$$
\lim _{\delta \rightarrow 0} \frac{1}{(2 \delta)^{\alpha+2}} \nu_{\alpha} \varphi^{-1}\left(S\left(\gamma_{0} \delta, \zeta\right)\right)=0
$$

uniformly in $\zeta \in \partial D$. Hence $\nu_{\alpha} \varphi^{-1}$ is a vanishing Carleson measure on $\mathbb{D}$. The proof of (4) $\Rightarrow(1)$ is similar to the proof of $(4) \Rightarrow(1)$ of Theorem 4.2. So we omit the details. 


\section{REFERENCES}

[1] S. Axler, 'Bergman spaces and their operators', in Surveys of some recent results in operator theory, Vol. 1, Pitman Research Notes in Math. 171 (Longman Science and Technology, Harlow, 1988), pp. 1-50.

[2] J. Cima and A. Matheson, 'Essential norms of a Composition operators and Aleksandrov Measures', Pacific J. Math. 179 (1997), 59-63.

[3] M. Hasumi and S. Kataoka, 'Remarks on Hardy-Orlicz classes', Arch. Math. 51 (1988), 455-463.

[4] B.D. MacCluer and J.H. Shapiro, 'Angular derivatives and compact composition operators on Hardy and Bergman spaces', Canad. J. Math. 38 (1986), 878-906.

[5] H.J. Schwartz, Composition operators on $H^{p}$, (Thesis) (University of Toledo, 1969).

[6] J.H. Shapiro and C. Sundberg, 'Compact composition operators on $L^{1}$ ', Proc. Amer. Math. Soc. 108 (1990), 443-449.

[7] J.H. Shapiro and P.D. Taylor, 'Compact,nuclear and Hilbert-Schmidt composition operators on $H^{2}$, Indiana Univ. Math. J. 23 (1973), 471-496.

[8] J.H. Shapiro, 'The essential norm of a composition operator', Ann. of Math. (2) 125 (1987), 375-404.

[9] J.H. Shapiro, Composition operators and classical function theory (Springer-Verlag, New York, 1993).

[10] S. Stevic, 'On generalised weighted Bergman spaces', Complex Var. Theory. Appl. 49 (2004), 109-124.

Department of Applied Physics and Mathematics Shri Mata Vaishno Devi University

Jammu-180006

India

e-mail: aksju_ 76@yahoo.com
Department of Mathematics University of Jammu Jammu-180006

India

e-mail: somdatt_ jammu@yahoo.co.in 